\title{
DE L'INTÉRÊT HEURISTIQUE DU CONCEPT DE « FANTÔME GÉOGRAPHIQUE » POUR PENSER LES RÉGIONALISATIONS CULTURELLES
}

\author{
Béatrice von Hirschhausen
}

Belin | «L'Espace géographique »

2017/2 Tome 46 | pages 106 à 125

ISSN 0046-2497

ISBN 9782410008654

Article disponible en ligne à l'adresse :

https://www.cairn.info/revue-espace-geographique-2017-2-page-106.htm

Distribution électronique Cairn.info pour Belin.

(C) Belin. Tous droits réservés pour tous pays.

La reproduction ou représentation de cet article, notamment par photocopie, n'est autorisée que dans les limites des conditions générales d'utilisation du site ou, le cas échéant, des conditions générales de la licence souscrite par votre établissement. Toute autre reproduction ou représentation, en tout ou partie, sous quelque forme et de quelque manière que ce soit, est interdite sauf accord préalable et écrit de l'éditeur, en dehors des cas prévus par la législation en vigueur en France. Il est précisé que son stockage dans une base de données est également interdit. 


\title{
De l'intérêt heuristique du concept de "fantôme géographique " pour penser les régionalisations culturelles
}

\author{
Béatrice VON HIRSCHHAUSEN \\ CNRS-UMr 8504 Géographie-cités \\ 13 rue du Four \\ F-75006 Paris \\ hb@cmb.hu-berlin.de
}

RÉSUMÉ . - Le concept de « fantôme géographique ", propose une réponse théorique à une série d'énigmes empiriques particulières, observables en Europe centrale et orientale. Les cas d'espèce dont il traite sont heuristiquement riches pour avancer sur la question de la production et de la reproduction dans la durée de différences entre des sociétés voisines et pour interroger plus généralement les phénomènes de différenciation géographique et de régionalisation à une échelle que les derniers développements de la géographie culturelle ont notoirement tendance à négliger, voire à récuser.

DÉCONSTRUCTIVISME, EUROPE, GÉOGRAPHIE CULTURELLE, GÉOHISTOIRE, IMAGINAIRE, STRUCTURE
ABSTRACT .- The heuristic interest of the concept of "phantom borders" in the understanding of cultural regionalisation.The concept of "phantom borders" offers a theoretical answer to a series of specific, empirical enigmas in Central and Eastern Europe. The individual cases addressed by this concept are heuristically rich in possible answers to the question of the long-term production and differences in neighboring societies. It also contributes to a more general analysis of the phenomena of geographic differentiation and regionalization at a scale that the latest developments in cultural geography notoriously tend to neglect and even reject.

DECONSTRUCTIVISM, EUROPE, CULTURAL GEOGRAPHY, GEO-HISTORY, GEOGRAPHICAL IMAGINATION, STRUCTURE e défi nous a été lancé par des - cartes thématiques figurant des données quantitatives: par des cartes électorales comme celle de la Pologne (Zarycki, 2000 et 2015; Rey et al., 2004; Grosfeld, Zhuravskaya, 2013) ou de l'Ukraine (Dessemontet, 2005 ; Löwis, 2015 ; voir également l'article de Sabine von Löwis dans ce même numéro) qui, à chaque rendez-vous électoral depuis un quart de siècle, laissent réapparaître avec une clarté stupéfiante les frontières des empires qui s'étaient partagé leurs territoires respectifs il y a plusieurs générations; question adressée aussi par des cartes des niveaux d'équipement des maisons rurales en 
Pologne ${ }^{1}$, dans les États postyougoslaves ou en Roumanie (Hirschhausen, 2015; voir aussi fig. 1) dont les villageois développent des stratégies de modernisation différentes selon que leur village fut, il y a plus d'un siècle, russe, ottoman, austro-hongrois ou prussien.

Nous avons nommé "frontières fantômes " ces traces laissées dans les sociétés contemporaines par des territorialités défuntes ${ }^{2}$. Du fantôme, elles tiennent leur caractère imprévisible: elles peuvent apparaître sur des cartes à l'occasion d'un rendez-vous électoral ou d'un recensement des logements et rester en revanche invisibles dans bien d'autres domaines; dans les exemples que nous venons de citer, rien par exemple ne signale leur trace quand on enquête sur la densité des abonnements téléphoniques ou bien sur les usages que font les internautes de Facebook ou de Instagram. À la différence de frontières invisibles (François, 1993) ou bien des frontières culturelles entre groupes ethniques (Boundaries)(Barth, 1969) qui ne sont

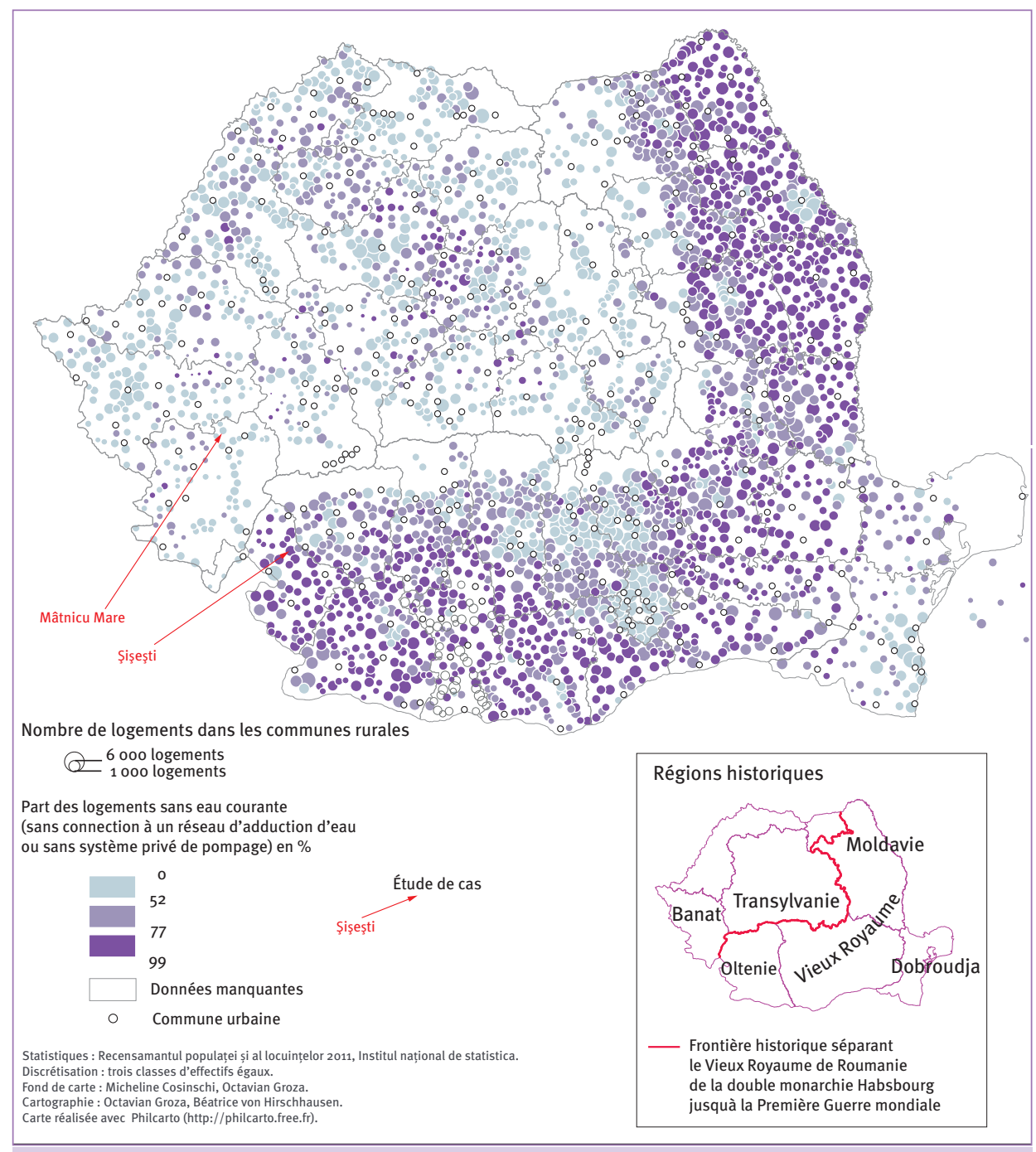

Fig. 1/ Les logements sans eau courante dans les campagnes roumaines en 2011
1. Voir la carte publiée par le journal en ligne polonais http://natemat.pl/, en 2014, à partir du recensement des logements de 2011 et qui laisse apparaître comme particulièrement peu dotés en équipements sanitaires les campagnes de l'ancien Royaume du Congrès (sous administration russe au cours d'un long $\mathrm{XIX}{ }^{\mathrm{e}}$ siècle de partage de la Pologne):

http://natemat.pl/108469,p olska-wschodnia-mieszkaw-domach-bez-lazienki-tamapa-zadziwilainternautow-ale-nie-jesttak-zle-jak-pokazuje

2. Le projet "Phantomgrenzen in Ostmitteleuropa " [les frontières fantômes en Europe centrale et orientale] a été développé dans le cadre d'un réseau de recherche financé entre 2011 et 2017 par le ministère fédéral allemand de

I'Enseignement et de la Recherche. Voir http://phantomgrenzen.eu/ 
pas davantage marquées au sol mais qui font fonction d'interface entre des communautés, régulent leurs échangent, font l'objet de renégociations permanentes sur les limites du franchissable et du transgressif, les frontières fantômes, quant à elles, ne freinent ni ne régulent les circulations; on les franchit sans transgression; elles tiennent moins de la frontière que de la discontinuité. Les sociétés locales peuvent même n'en avoir qu'une conscience confuse.

Ces cas d'espèce nous semblent heuristiquement riches pour avancer sur la question de la production et de la reproduction dans la durée de différences «culturelles » entre des sociétés voisines et pour interroger plus généralement les phénomènes de différenciation géographique à une échelle d'analyse régionale que les derniers développements de la géographie culturelle francophone ont notoirement tendance à négliger. Nous commencerons par éclairer ce qui fait de la question des "géographies fantômes " et des «frontières fantômes » un objet heuristique au regard des débats théoriques portant sur les découpages régionaux avant de descendre au niveau de l'analyse d'une étude de cas. Puis nous chercherons à remonter vers la formalisation d'un concept opératoire permettant de penser la figure particulière des géographies fantômes et, au-delà, de rénover la question de la construction dans la durée d'aires culturelles.

\section{Les enjeux théoriques d'une énigme empirique}

\section{Par-delà la fascination visuelle}

On pourrait bien sûr ranger les frontières fantômes au rang des phénomènes étranges, dotés d'un fort pouvoir de fascination visuelle, et bons pour des cabinets de curiosités. Ce serait toutefois ignorer leur potentiel heuristique au regard des débats sur la manière d'aborder les aires culturelles et les longues durées géohistoriques. Ce serait aussi négliger leur potentiel politique: comme les fantômes dans le spiritisme, elles peuvent se voir convoquées par des acteurs politiques qui cherchent à les " réveiller " dans les mémoires collectives à la faveur de crises politiques; elles peuvent être mobilisées dans les discours identitaires et participer à la construction de "communautés imaginées" (Anderson, 1996). La séparation de la République tchèque de la Slovaquie en 1992, plus dramatiquement, la guerre de Yougoslavie il y a vingt-cinq ans ou le conflit en Ukraine aujourd'hui, sont là pour rappeler comment des frontières fantômes, une fois instrumentalisées par des acteurs politiques, peuvent, d'un état inoffensif, développer des pouvoirs de sape pour les États qu'elles traversent. La difficulté et l'enjeu à la fois théorique et empirique d'un tel objet consistent justement à ne pas participer à la réification des fantômes géographiques, et à en élaborer une lecture qui soit capable d'en saisir les mécanismes de construction dans l'ensemble du champ social, par-delà le seul registre de leur production discursive.

Cela suppose que l'on ait au préalable pris la mesure des enjeux théoriques qui traversent cet objet. En effet, la question des géographies fantômes doit être resituée au regard de deux débats qui comportent de nombreux parallélismes, d'une part dans le cadre de la critique postcoloniale, autour de la définition des aires culturelles et d'autre part, dans le champ de la géographie culturelle et de ses différentes déclinaisons dans les espaces anglophone, germanophone et francophone sur la pertinence des approches régionales. 


\section{Une naïveté impossible}

Les fantômes que laissent voir les cartes ne sauraient se soustraire aux controverses développées autour de la question des aires culturelles. Dans les cas que nous décrivons, ils renvoient à des territorialités impériales qui ont durablement marqué la construction des sociétés: l'empire russe puis sa déclinaison soviétique, l'Empire ottoman ou l'empire des Habsbourg ont, sur de longues périodes, organisé localement la distribution des pouvoirs, établi des régimes de gouvernance, diffusé des schémas esthétiques, façonné les territoires. Les paysages urbains, les morphologies villageoises ou foncières, les compositions ethniques ou linguistiques en gardent la trace. Les définitions de l'Europe centrale, des Balkans ou de l'Europe orientale sont justement calées sur ces référents impériaux et attachées, dans les représentations des Européens, à des modèles de société et, dans leurs sillages, à des systèmes de valeurs tout comme à des paysages. Cette coïncidence, toujours opérante dans les représentations communes des Européens, entre espaces, histoire et cultures continue d'organiser les savoirs sur les sociétés non occidentales. Du temps de la Guerre froide, c'était les frontières de la domination soviétique qui définissaient les limites des études est-européennes. Depuis les années 1990, les aires géographiques découpées pour définir les aires d'étude de chaires universitaires ou de centres de recherches ont glissé vers une distinction d'ensembles " culturels " ou " civilisationnels " ancrés dans de plus longues durées historiques.

Ces découpages dans les représentations comme dans l'organisation des savoirs ont parallèlement fait l'objet d'une puissante critique, les accusant de donner les aires culturelles pour un fait établi, de les traiter comme des "containers" (selon le terme anglophone) dont il faudrait mettre à jour la spécificité et les traits "homogènes " en passant sous silence leur dimension largement construite et en négligeant les superpositions, réseaux, circulations, échanges, qui font la trame transrégionale d'un monde globalisé (pour une synthèse de cette critique produite en langues allemande et anglaise voir Bachmann-Medick, 2006, p. 292-302). À partir en quête des traces de fantômes impériaux, on peut être rapidement accusé de conforter ces découpages, d'en réifier les contenus en marginalisant d'autres compositions spatiales plus efficaces. On cèderait à une forme de "régionalisme méthodologique " qui postulerait a priori l'unité de l'aire régionale étudiée, qui en naturaliserait le découpage et qui rendrait aveugle aux tissages transnationaux, transrégionaux, transculturels des sociétés contemporaines et à leur inscription dans des dépendances globales, de types migratoire, économique et symbolique. Plus gravement encore, en cherchant à repérer et à qualifier les différences de part et d'autres des frontières fantômes, on conforterait la stigmatisation de régions considérées à l'aune de leur retard par rapport à l'étalon démocratique ou économique de régions voisines. Ainsi, par exemple, la carte des équipements domestiques en Roumanie, pourrait être lue comme une description de "l'archaïsme " des anciennes provinces du Vieux Royaume au regard des provinces " centre-européennes " sorties quant à elles de la matrice impériale des Habsbourg et les renvoyer à un "balkanisme " stigmatisé. L'analyse scientifique ne ferait alors que valider une "carte mentale " qui figure l'Europe centrale en "Occident kidnappé» (Kundera, 1983) doté du même héritage culturel que l'Europe de l'Ouest et " destiné " au même développement, même si la capture soviétique l'en avait un temps privé, et qui renvoie à l'inverse les périphéries balkaniques et orientales à leur sous-développement quasi "atavique ». On ne saurait donc aborder les fantômes géographiques sans clarifier sa position théorique au regard de ces critiques.
3. Nous employons ici l'expression de " carte mentale ", reprise à l'expression anglaise de " mental map " ou " mental mapping " qui s'est imposée chez les historiens de langue allemande pour désigner les charges normatives et les valeurs associées aux différentes parties du monde,

dans les représentations communes ou dans les écrits savants (Schenk, 2002). 
4. Les auteurs parlent à propos de la région de "Fallstricke, räumelnden' Denkens", de "piège de la pensée spatialisante " (p. 3).

5. Dans la géographie allemande, la critique s'était adressée en premier lieu à la Landschaftsgeographie mais aussi à la Länderkunde élaborée dans de la première moitié du $\mathrm{xx}^{\mathrm{e}}$ siècle à partir d'une réception affadie des travaux de Alfred Hettner, alors dépouillés de sa perspective critique, et qui tendait à considérer les régions comme des " containers" spatiaux dont il faudrait de manière tautologique mettre à jour et décrire les caractères (Schultz, 1980).

6. Benno Werlen (1995a, p. 6) parle à propos d'une géographie centrée sur l'espace de " bloße Geographie der Dinge ".
Du côté de la géographie, la montée en puissance des questionnements culturels s'est accompagnée d'un recul des études à échelle moyenne et d'un scepticisme accru à l'égard des aires culturelles. La question des frontières fantômes ne saurait ainsi échapper à la méfiance théorique radicale développée par les géographes angloaméricains, puis de langue allemande à l'égard du "piège réaliste " des approches régionales (Glasze et al., 2014) ${ }^{4}$. À la première critique des années 1960 et $1970^{5}$ qui avait conduit comme en France à l'adoption, dans le sillage de la science régionale, d'un paradigme quantitativiste et modélisateur récusant le paradigme classique et idiosyncrasique de la région, a fait suite une seconde critique, élaborée vingt ans plus tard, sous l'effet d'un puissant tournant culturel, mettant notamment en cause l'arbitraire des découpages régionaux produits par les savoirs géographiques, et leur inadéquation à la pensée d'un monde globalisé. La région s'est dès lors affirmée, non comme un donné dont on pourrait définir les contours avant d'en examiner le contenu, les fonctionnements ou des dynamiques identitaires mais comme une construction dont il faudrait repérer les différents moments de cristallisation en remettant l'intentionnalité des acteurs et leur agir quotidien au centre de l'investigation (pour ce débat en Allemagne sur la nature de la région voir notamment: Blotevogel, 1996; Weichhart, 1996; Schultz, 1997). Benno Werlen (1995b et 2004) conteste une quelconque capacité de l'espace, dans le monde contemporain globalisé, à expliquer les réalités culturelles. Il préconise le passage d'une géographie sociale à une science sociale géographique qui ne soit plus centrée sur les régions et sur les découpages de "classes spatiales ", et qui parte à l'inverse des processus quotidiens de régionalisation du social (Werlen 1995a, p. 4-5). Certains auteurs en viennent à considérer la région comme une construction discursive, dont il faudrait décortiquer le processus d'élaboration dans les écrits scientifiques, dans la fabrication des cartes, dans les propos des politiques ou dans les représentations médiatiques (pour une synthèse de cette évolution voir Wardenga, Miggelbrink, 1998). Dans ce contexte, notre comptage des robinets d'eau dans les villages roumains de part et d'autre d'une ancienne frontière impériale pourrait passer, au mieux, pour une démarche à contre-pied, au pire pour un retour irréfléchi sur les vieux schémas d'analyse régionale. Il nous faudra donc préciser en quoi il ne s'agit pas pour nous de produire une "géographie plate des choses $\|^{6}$ mais bien de questionner la construction des différences régionales dans la durée.

Même si la géographie culturelle française s'est montrée sur le sujet beaucoup moins polémique ou prompte au débat, le défi théorique n'y est sans doute pas moindre. Elle n'a certes pas connu, jusqu'à ce jour, pareille mise en cause frontale de la pertinence des cadres d'analyse régionaux; elle ne s'en est pas moins détournée des questionnements sur la production des différences culturelles aux échelles moyennes. Une fois achevée en 1996, la monumentale édition de la Géographie Universelle (Brunet, 1990-1996) qui constituait l'aboutissement d'un vaste et ambitieux projet intellectuel, porté par la génération de la "nouvelle géographie » des années 1970 et par les meilleurs spécialistes de la géographie régionale francophone, le questionnement sur les découpages du monde a connu un spectaculaire effacement des agendas de la recherche dans la discipline. Sur la région qui nous intéresse ici, la voie ouverte par Violette Rey pour questionner les spécificités centre et sud-est-européennes sur un mode très original en partant de l'expérience historique particulière à cet espace " entre-deux " et du rapport au temps et à l'histoire de ses habitants (Rey, 1995; Rey et al., 1996) n'a finalement pas suscité de véritable débat. À une autre échelle, le travail 
de Christian Grataloup (1996) sur la géohistoire est demeuré solitaire. Si les découpages régionaux structurent encore l'organisation des enseignements universitaires de "géographie régionale " ou les programmes des concours de l'enseignement, ils ne sont plus guère problématisés et le terme de "région ", n'apparaît plus dans la littérature scientifique que sous une forme générique et vidée de ses enjeux théoriques: les débats se sont déplacés vers les notions de territoire et de territorialisation, en remettant les acteurs et la construction de leurs identités culturelles et territoriales au centre du questionnement (Debarbieux, 2006), mais en évacuant du coup le problème des particularités régionales et d'une pensée géographique du monde aux échelles moyennes. C'est malheureusement au moment où les géographes se détournaient de la question des longues durées géohistoriques que la thèse du " choc des civilisations " connaissait son grand succès médiatique. Rares sont alors les auteurs en géographie qui ont cherché à relever le défi pourtant grave et pressant que lançait le schéma d'interprétation développé par Huntington ${ }^{7}$. La question posée par nos cartes nous apparait paradoxalement, dans ce contexte épistémologique, aussi urgente que difficilement audible. Là aussi, il faudra en expliciter la posture et l'actualité théorique.

\section{La polarisation des postures de recherche entre approches structurales et déconstructivistes - échapper à l'alternative}

Dans le contexte que nous venons de décrire, s'est opérée une forme de polarisation des postures de recherche. D'un côté des travaux continuent de poser l'existence de structures (économiques, sociales, politiques, culturelles) capables de composer le réel et qui considèrent que l'espace géographique acquiert en retour une capacité à organiser le social. De l'autre s'affirment des postures déconstructivistes qui traquent dans les sphères du langage, des représentations et de la production des savoirs, les processus de fabrication des imaginaires du réel et des géographies mentales des sociétés, considérés comme seuls niveaux accessibles à un savoir forcément tributaire des catégories langagières. Les deux postures n'ont cessé au cours des dernières décennies de diverger et elles tendent à se développer séparément en s'ignorant réciproquement. Les débats sont très actifs à l'intérieur des deux camps mais peu nombreux entre eux (Besse, 2004; collectif, 2004). Les postures déconstructivistes ont pour une part forcé les premières à davantage de réflexivité et débouché sur l'élaboration d'un réalisme critique sans toutefois en modifier les prémisses. D'une certaine manière, chacun est sommé de choisir son camp et les travaux qui se sont penchés récemment sur les héritages impériaux dans les sociétés centre-est européennes, obéissent à cette partition.

Ces recherches sont moins le fait de géographes (qui tendent, nous l'avons dit, à déserter la question des différences régionales) que d'autres disciplines. Du côté des approches structurales, on trouve les travaux notamment anglo-américains d'économie politique ou de science politique qui, s'adossant à la théorie de "Path path dependency " (Pierson, 2004), à celle du "social capital " collectif (Putnam, 1993), ou au concept d'héritage ("legacy "), cherchent à remonter aux origines des divergences électorales ou des écarts entre les pratiques économiques à des niveaux régionaux (Stark, Bruszt, 1998; Murgescu, 2010). Certains travaux postulent l'existence de différences géographiques relativement stables, d'ordre culturel ou civilisationnel, établies à des périodes plus ou moins éloignées et dont ils cherchent à repérer les prémisses ou les voies de reproduction dans la durée. C'est le cas par exemple de l'analyse conduite par une équipe internationale et interdisciplinaire d'économistes et d'historiens sur les régimes
7. L'exception doit être faite ici du dossier thématique très interdisciplinaire, coordonné en 2013 par Georges Prévélakis dans la revue Anatoli sur "Huntington, 20 ans après "; mais il n'a connu finalement que peu de relais dans le champ de la géographie. 
8. C'est d'ailleurs cette lecture polarisée de l'est et de l'ouest qui structurait une partie des discours entendus sur Maïdan en 2013-2014 et I'interprétation que fit la presse internationale de la crise ukrainienne. La lecture russe, quant à elle, dans une version inversée de la polarisation géographique, renvoyait l'ouest ukrainien à son penchant fasciste, auquel l'est devait opposer sa résistance morale et politique. de confiance et de corruption prévalant dans les différentes régions du Monténégro, de Pologne, de Roumanie, de Serbie, d'Ukraine, de part et d'autre de l'ancienne frontière orientale et méridionale de l'Empire des Habsbourg (Becker et al., 2016). Elle compare les mesures faites pour un panel d'enquêtes individuelles menées en 2006 dans un grand nombre de localités de l'Europe postsocialiste, sur la confiance que les habitants font à leurs administrations locales (justice et police locales) et observe un fort différentiel entre les anciennes régions de la double monarchie et celles qui sont issues de l'empire russe ou de l'empire ottoman. Sans se prononcer formellement sur les modalités de transmission des normes et des pratiques au sein de sociétés qui ont par ailleurs connu au cours du dernier siècle de puissants déplacements de populations, les auteurs concluent à l'existence de canaux d'apprentissage territoriaux, qui auraient véhiculé, au sein des administrations et des communautés locales, des formes d'éthique citoyenne, mises en place avec l'instauration d'un État bureaucratique impérial au cours du $\mathrm{XIX}^{\mathrm{e}}$ siècle et qui auraient été capables de perdurer sous les régimes socialistes. D'autres travaux de même type portent sur le colportage dans la durée des attachements pro-européens ou pro-russes de part et d'autre de l'ancienne frontière habsbourgeoise en Ukraine (Peisakhin, 2013 et 2015) ou interrogent la conformité de la carte des résistances à l'hégémonie soviétique avec celle des scolarisations de masse présoviétiques (Darden, Grzymala-Busse, 2006).

Dans l'autre "camp ", des travaux, menés pour l'essentiel par des historiens dans le sillage des Post-colonial Studies et de l'analyse d'Edward Said (1980) sur l'orientalisme, n'ont pas manqué de relever que les distinctions opérées entre les héritages impériaux font écho aux représentations de l'ouest et de l'est qui structurent les imaginaires de l'espace européen. À l'ouest les représentations prêtent aujourd'hui la modernité, l'autonomie de l'individu, le sens de l'initiative économique et de la démocratie, à l'est, le collectivisme, la corruption, et le penchant pour des régimes autoritaires $^{8}$. L'analyse du déplacement progressif, au tournant du XVIII et du XIX ${ }^{\mathrm{e}}$ siècles, de l'axe des valeurs " civilisation-barbarie ", d'une orientation méridienne sud-nord vers une orientation latitudinale ouest-est sur les cartes mentales européennes a fait l'objet de plusieurs travaux critiques qui ont fait date (Lemberg, 1985; Wolff, 1994). Une abondante littérature dessine depuis une trentaine d'années une nouvelle historiographie des "mental maps " des Européens (Schenk, 2002), occupée à historiciser les découpages géographiques produits par les discours savants et politiques (Grataloup, 2011), à repérer les valeurs morales qui leur sont attachées et à dénoncer les ambitions hégémoniques qu'ils justifient (Kraft, 2015). L'historienne américaine Maria Todorova (2011) s'en est notamment prise à la réactualisation, au cours des années 1980, de la catégorie d'"Europe centrale " par les historiens et intellectuels de Hongrie, Pologne ou Tchécoslovaquie, qui avaient cherché, pour mieux s'arracher à l'oubli des occidentaux et se distinguer de l'«Europe de l'Est ", à afficher la proximité de leurs héritages culturels avec l'Occident; elle y voit un moment clé de la consolidation, dans les représentations, d'une image "stigmatisée » de l'altérité balkanique.

Le débat entre ces deux manières opposées, structurale ou déconstructiviste, de penser les différences entre les régions historiques de l'Europe a connu dans l'ensemble de la communauté des historiens de langue allemande un écho particulier, à travers une série de quatre articles publiés entre 1999 et 2003 et opposant Holm Sundhaussen et Maria Todorova notamment dans la grande revue d'histoire, Geschichte und Gesellschaft (Sundhaussen, 1999, 2003; Todorova, 2002, 2003, 2007). Holm Sundhaussen, 
historien très reconnu de la péninsule Balkanique, y défend la possibilité de construire des catégories régionales, certes fluides et dont les frontières sont soumises à redéfinition au fil des développements historiques, mais fondées sur un ensemble de traits régionaux partagés. Il identifie pour les Balkans, un faisceau ("cluster ") de huit caractères sociaux, politiques et économiques, caractéristiques de la région sur une longue durée, et formés au fil des expériences historiques byzantine, ottomane et post ottomane ${ }^{9}$. Maria Todorova reproche à sa démarche d'" exotiser " et d'essentialiser des entités territoriales trop statiques et in fine de ne faire que prolonger par une argumentation scientifique les clichés balkaniques et et les imaginaires de l'Europe inventés par les publicistes et les discours politiques depuis le XIX ${ }^{e}$ siècle. Cette dispute et sa résonnance dans le champ des études historiques, bien au-delà de la sphère des spécialistes du sudest européen, n'a toutefois pas débouché sur l'ouverture de nouvelles pistes de recherche. Et pour cause: l'alternative épistémologique ainsi posée tient en effet, selon nous, de l'impasse. Aucune des deux postures structurale ou déconstructiviste qui s'excluent réciproquement, et qui mettent les différences régionales, pour l'une au compte de processus cumulatifs dont les acteurs seraient prisonniers, et pour l'autre au compte de la puissance des discours et de représentations hégémoniques, n'est en mesure d'épuiser à elle seule le problème de la construction des différences géoculturelles. Cette incapacité des approches canoniques à expliquer de manière satisfaisante les fantômes de nos cartes et plus généralement la construction historique de différences culturelles dans l'espace, en fait des quasi-objets de laboratoire appellant une recherche inventive. C'est à la quête de nouveaux moyens théoriques pour sortir de l'aporie et éclairer l'énigme de nos cartes qu'il faut s'employer.

\section{Repartir de l'empirie: les choix et les capacités d'agir des acteurs locaux}

Pour comprendre ce qui se joue derrière l'apparition cartographique de fantômes d'empires dans les sociétés contemporaines, nous avons pris le parti de quitter les postures en surplomb qui caractérisent les travaux précédents et de descendre aux niveaux locaux des acteurs, à l'horizon de leurs regards et de leurs choix. Des enquêtes menées dans des villages situés de part et d'autres de tracés repérés sur nos cartes ont permis de questionner leurs habitants, sur leur agir quotidien, leur compréhension du politique ou de la modernité et leur manière de se situer au sein de leur espace national respectif. Nous avons choisi d'interroger conjointement et les effets potentiels de "structures " et les "imaginaires " de l'espace en prêtant une attention particulière à la manière dont ces deux dimensions se nouent au cœur de l'expérience individuelle et collective des villageois. Pour être cohérente, la démarche devra aussi s'adosser à une analyse des données disponibles à l'échelon régional. À ce moment de notre recherche, nous nous arrêterons à la confrontation de deux analyses conduites à une micro-échelle mobilisées dans une visée comparative et régionale.

\section{Étude du cas roumain}

À la différence d'autres études de cas conduites dans le cadre de la recherche collective ${ }^{10}$, les deux villages que nous étudions en Roumanie ne sont pas contigus. La frontière impériale a ici suivi la ligne de crête des Carpates et les faibles densités des sommets du massif. Les échanges et circulations avant 1914 n'étaient certes pas
9. Holm Sundhaussen compte au rang de ces huit caractères: 1) l'instabilité du peuplement et la grande fragmentation territoriale entre groupes ethniques; 2) la perte, puis la réception tardive de l'héritage antique; 3) I'héritage orthodoxe byzantin; 4) I'héritage islamique ottoman;

5) un certain " retard" socio-économique à

l'époque moderne: 6) une formation de l'État et de la Nation traversée par de vives concurrences entre les peuples et marquée par un mélange des arguments modernes et de mythes du passé balkanique;

7) une culture politique marquée par les mentalités patriarcales et les mythes; 8) l'interventionnisme récurrent des grandes puissances.

10. Voir par exemple I'article de Sabine von Löwis dans ce même numéro. 
inexistants ou seulement le privilège des élites; ils étaient également le fait des bergers transhumants, des contrebandiers ou de ces rituels rendez-vous annuels ("nedei») qui réunissaient, le temps d'une fête, sur les hautes estives, les communautés villageoises des deux versants, et où se nouaient les échanges commerciaux et les alliances matrimoniales transfrontalières. En dépit de ces circulations, la frontière politique qui partageait l'espace jusqu'à la fin de la Première Guerre mondiale a correspondu de fait à une discontinuité du peuplement toujours bien repérable sur la carte de la figure 1. Les points de passage qui remontent les vallées et franchissent les cols sont encore aujourd'hui relativement peu nombreux. Nous avons en conséquence conduit la comparaison en travaillant sur des villages de taille et de structure comparables, situés de part et d'autre de l'ancienne frontière, et séparés de $100 \mathrm{~km}$ à vol d'oiseau et de $160 \mathrm{~km}$ par la route. Le village de Mâtnicu Mare est situé à l'ouest, dans la région du Banat, sur une terrasse de la vallée du Timis. Il a gardé le plan géométrique de ces villages de colons installés au XVIII ${ }^{\mathrm{e}}$ siècle par l'Empire des Habsbourg sur les terres reconquises avec la paix de Passarowitz, pour sécuriser ces nouvelles marches qui le séparaient des Ottomans. Les maisons, imposantes et jointives, soulignent le front des rues rectilignes. Les cours paysannes demeurent cachées derrière de hauts porches. L'autre village d'étude, Şişeşti, est situé de l'autre côté des Carpates, à l'est de l'ancienne frontière. Il est d'une tout autre facture: les maisons s'égrènent le long des chemins, derrière leurs palissades de bois ou leurs nouvelles clôtures de fer forgé.

Des deux côtés des Carpates, les sociétés villageoises ont connu la même collectivisation des terres à la fin des années 1950, la lente modernisation de la vie rurale: l'électrification dans les années 1960, l'essor de l'emploi industriel dans les années 1970 et le développement d'une double activité des familles qui laissait les femmes travailler pour la coopérative agricole au village et envoyait les hommes dans les usines ou les mines des petites villes voisines. Des deux côtés, la même dégradation des conditions matérielles au long des années 1980 marquées par les pénuries et l'arbitraire du fonctionnement des coopératives. La rudesse des conditions de vie nourrissait l'exode des plus jeunes et des plus qualifiés vers les villes et bassins industriels du pays. Des deux côtés, les familles des ouvriers-paysans avaient tout investi dans la construction ou la modernisation de leurs maisons, bien que la pénurie drastique de matériaux de construction et de biens d'équipement au cours de la dernière décennie $\mathrm{du}$ régime ait fortement limité leurs projets: seules quelques familles avaient pu installer une pompe dans leur puits et se doter de l'eau courante dans la cuisine, d'une salle de bain, d'une machine à laver, voire de sanitaires. Au début des années 1990, après une brusque décollectivisation suivie d'une profonde désindustrialisation qui plongea la plupart des familles dans un grand désarroi économique, les niveaux d'équipement domestique sont restés faibles des deux côtés.

C'est à partir des années 2000 que les statistiques des recensements de 2002 puis 2011 laissent deviner une évolution radicalement divergente des stratégies des familles en matière d'équipement domestique, en dépit de niveaux de vie très comparables (Rey et al., 2000, 2007). Dans la commune banataine où est situé Mâtnicu Mare, le taux des logements dotés d'eau courante, encore insignifiant en 1992, passe à 13,9\% en 2002, puis bondit à 78,8\% au recensement de 2011. Dans la commune olténienne de Şişeşti, il passe au même moment à $1 \%$ puis à $4,4 \%$. Les enquêtes en village montrent que la modernisation des téléviseurs, les abonnements téléphoniques, l'achat d'ordinateurs et les accès à internet ne connaissent pas la même dissymétrie. 
Des deux côtés, ces indicateurs connaissent une hausse remarquable. On n'a donc pas ici affaire à une différence entre des " archaïques " et des "modernes »; il s'agit plutôt de manières différentes de jauger les priorités.

Impossible de restituer ici le détail de l'analyse (Hirschhausen, 2015). Nous nous contentons de relever quelques faits stylisés. On peut repérer des traits structurels qui donnent une partie des clés de l'explication. La nature du bâti villageois par exemple: les grosses bâtisses de brique des villages du Banat autorisent une modernisation plus rapide; les pièces sont nombreuses et permettent d'y créer des équipements sanitaires. À l'inverse les maisons traditionnelles de bois et torchis à Şişeşti sont de taille beaucoup plus modeste; quand leurs moyens le leur permettaient, les habitants ont commencé à construire en brique à partir des années 1970 et surtout 1980; ce matériau y est d'usage beaucoup plus rare; les familles qui veulent moderniser leur équipement sanitaire, dans ce village d'Olténie, doivent en général construire une annexe, adjoindre de nouveaux corps de bâtiments voire bâtir une nouvelle maison: c'est à la fois long et coûteux. Le niveau moyen de qualification et les importances respectives des élites de village pourront aussi constituer des indicateurs de type structurel portant sur la population cette fois-ci. Les intellectuels de village et les personnes dotées d'un diplôme du supérieur sont proportionnellement plus nombreux dans les villages du Banat. Ce sont ces personnalités qui ont le plus souvent lancé le mouvement de modernisation partout dans les campagnes roumaines, avec cependant un décalage temporel de plus de quinze à vingt ans selon les régions: les premières pompes furent installées dans la commune de Mâtnicu Mare au début des années 1970 par les médecins et enseignants. Celles de Şişeşti, à la fin des années 1980 par les paysans mineurs, puis au début des années 1990 par les intellectuels de village. Si l'on place ces causalités de type structurel au regard des représentations villageoises, on constate que les habitants eux-mêmes invoquent en partie ces éléments factuels quand ils cherchent à se situer les uns par rapport aux autres. Les villageois de Şişești disent leur admiration pour les grosses bâtisses et pour l'ordonnancement régulier et imposant des villages de la région voisine qu'ils connaissent par ouï-dire, par les images médiatiques ou parce qu'ils y ont eux-mêmes séjourné et travaillé. Les habitants de Mâtnicu Mare de leur côté soulignent volontiers la robustesse de leur habitat, leur fierté devant leurs élites, la réputation de leur ardeur au travail et surtout au travail bien fait. Des deux côtés, tous éclairent et situent ces faits matériels et sociaux au regard d'un imaginaire collectif qui " distingue » le Banat, et l'identifie dans les représentations collectives de la Roumanie comme plus "occidental " et "plus civilisé ". Pour chacun, cela va de soi. Vécu comme un état de fait, cet imaginaire des espaces qui inscrit l'ancien espace habsbourgeois dans le grand récit du Progrès et de la Civilisation occidentale "oblige " (au sens où " noblesse oblige ") les villageois de ce côtéci des Carpates. Quand on les interroge sur leur habitat, ils font la narration de leur marche vers la modernisation et des investissements successifs, consentis pour améliorer l'isolation, le chauffage, l'équipement domestique, etc. Le moteur de l'agir des habitants du côté olténien, dans le village de Şişeşti, semble en revanche fonctionner sur un autre régime: l'accès à la "modernité " domestique y est placée non sous le signe de l'accumulation et de la transformation de l'existant mais sous celui de la rupture avec les modes de vie locaux et de l'importation de modèles et d'objets extérieurs. La profonde intériorisation des "mental maps " de l'Europe est ici à l'œuvre. La construction de nouvelles maisons selon de nouveaux codes architecturaux dans 
les campagnes d'Olténie constitue la manifestation la plus visible de ce désir de rupture avec les modèles traditionnels.

\section{Essais d'interprétation}

On remarque que nous avons renoncé dans l'analyse aux termes de "culture " et de " civilisation". Non pas que ne soient pas en jeu des processus de type culturel. L'important est cependant ici de ne pas nommer des aires culturelles ou civilisationnelles prédéfinies dont il s'agirait de reconstituer la trajectoire dans une perspective génétique; il s'agit plutôt de comprendre les processus à l'œuvre, mouvants, toujours susceptibles de redéfinition.

Dans le Banat, il s'agit de renouer, après l'épisode malheureux de la domination soviétique, avec le "destin géographique " d'une région considérée, par les habitants eux-mêmes, comme intrinsèquement "moderne " et vouée de longue date au développement. La ressemblance architecturale des villes et des villages avec ceux de l'Europe des Habsbourg, le baroque des bâtiments civils et religieux, mais aussi le tissage serré des sociétés urbaines et villageoises, les confortent dans cette vision de l'histoire régionale. Et cette foi partagée guide les choix des habitants, leurs investissements dans l'amélioration de leur habitat, comme d'ailleurs dans la modernisation de leur outillage agricole ou dans la formation professionnelle ou universitaire de leurs enfants. "Si il n'y avait pas eu le communisme, nous serions aujourd'hui au niveau de l'Allemagne ", entend-on souvent dire dans ces villages. Ici la foi dans l'avenir est articulée à la confiance en son propre passé. Cette dernière donne l'assurance de pouvoir s'adosser aux solides bâtisses construites par les générations précédentes. On peut " rattraper» le temps perdu en prenant appui sur l'héritage.

En Olténie, rien de comparable. Les villageois ne peuvent recourir à la même prophétie et le présent postsocialiste, marqué au sceau d'une brutale désindustrialisation, apparait trop peu intelligible pour permettre un pronostic optimiste sur l'avenir et pour justifier des stratégies parcimonieuses d'avancée à petits pas, via l'amendement de l'existant. La hiérarchie des choix se joue alors sur un autre registre et sur la base d'un passé en partie récusé qui appelle la rupture. À défaut de pouvoir accomplir matériellement, d'un coup, le grand saut vers un mode de vie "moderne » que leur confèrerait une nouvelle maison construite sur le modèle d'une "villa " à étage, dotée de toutes les commodités, la grande majorité des villageois rencontrés dans la commune de Şişeşti, commencent par se ménager un accès d'ordre symbolique à un avenir plus confortable. Ils préfèrent par exemple investir dans un nouveau portail, forgé sur un modèle inspiré des telenovelas qui passent en boucle sur les chaînes des bouquets numériques auxquelles ils sont abonnés, et acquérir ainsi une forme de promesse de ce confort futur, plutôt que de bricoler une pompe dans leur puits et un robinet dans leur cuisine qu'ils jugent d'un autre âge et inappropriée à cette modernité. C'est une manière pour eux de convoquer la rupture avec le passé des maisons paysannes vécues comme anachroniques et

11. Vintilă Mihăilescu propose de son côté, dans sa belle analyse de la " domestication de la modernité " dans les campagnes roumaines, une interprétation très voisine (Mihăilescu, 2011 et 2014). d'accéder d'emblée à une nouvelle esthétique, associée à la prospérité espérée. ${ }^{11}$ La position de l'héritage territorial olténien hors du grand récit modernisateur, disqualifie en quelque sorte son patrimoine bâti pour toute entreprise d'amendement progressif. Les nouveaux médias (abonnement au câble, accès à internet, inscription sur Facebook, téléphonie mobile, téléviseurs à écran plat, etc.) donnent un accès par bribe - duquel nul ne saurait se passer - à une réalité mondialisée, et coexistent sans contradiction avec l'univers matériel des maisons paysannes et des allées et venues quotidiennes au puits de 
la cour. À chacun de ces deux univers matériels correspondent deux régimes temporels disjoints qui ordonnent de manière quasi autonome deux échelles de priorités.

C'est à la manière dont se coconstruisent, à différentes échelles, les faits structurels (jusque dans leur matérialité) et les imaginaires de l'espace et de l'histoire que nous avons consacré l'essentiel de nos efforts empiriques et théoriques afin de "dénaturaliser " cet état de fait et d'en restituer la dynamique à la fois historique et "performative $»^{12}$. En effet, la construction des différences entre les deux régions apparaît historique, au sens où l'on peut restituer différents processus d'accumulation durables dans les espaces matériels et sociaux. Elle est aussi de nature "performative " au sens où les multiples choix et propos quotidiens des acteurs locaux actualisent en permanence dans la sphère matérielle et sociale une carte mentale qui promet le progrès à l'Europe centrale et le retard à l'Europe balkanique. Les habitants tendent à se conformer aux "destins " que les imaginaires assignent aux territoires. Ils s'y réfèrent pour donner sens à leur expérience, pour identifier les ressources du passé dont ils peuvent jouer et pour faire leurs choix en direction du futur.

\section{Contribution à une théorie des aires culturelles}

\section{La nature ambivalente du fantôme}

Le fantôme, lit-on dans Le Robert, est à la fois "l'apparition " d'un être disparu et " une fantasmagorie ». En d'autres termes, on ne sait jamais très bien, en présence d'un fantôme, ce qui procède du défunt (de sa corporéité et de son histoire) ou d'une projection de l'imaginaire de ses témoins oculaires dans l'ordre des vivants. C'est du côté de cette double nature que nous cherchons à tirer la métaphore afin de penser des dynamiques à l'œuvre particulièrement ambivalentes.

La rémanence des empires morts dans la géographie des pratiques passe en effet en partie par les corps des acteurs, par leurs gestes, leurs manières de dire et de faire, transmis dans les habitus, les normes et les usages sociaux; elle passe aussi par les paysages, par les structures foncières et les documents cadastraux, les morphologies villageoises et les architectures, les systèmes de peuplement et les réseaux d'infrastructure. "Le passé - écrivait Henri Lefebvre - a laissé ses traces, ses inscriptions, écriture du temps. Mais cet espace est toujours, aujourd'hui comme jadis, un espace présent, donné comme un tout actuel, avec ses liaisons et connexions en acte. De sorte que la production et le produit se présentent comme deux côtés inséparables et non comme deux représentations séparables" (Lefebvre, 1974, p. 47) ${ }^{13}$. Une part de ces logiques de (re)production dans la durée demeure inconsciente et procède de ces « connexions en actes " inscrites spatialement dans le rapport des êtres et des choses et qui réactualisent l'ordre social et matériel dont elles émanent, au moment même où elles ont lieu. Ces logiques peuvent être, dans certains cas, conscientisées par les acteurs régionaux qui savent en mobiliser la ressource: acteurs et choses composent alors des agencements qui "font territoire " (comme on dit parfois "font société 》) pour des durées variables dans un double mouvement de composition de l'espace et de production du sens. Ce que Bernard Debarbieux appelle le «paradigme territorial» (2006, p. 346 sq.) tel qu'il a été théorisé par certains auteurs comme Jean-Luc Piveteau, permet de concevoir ces dynamiques conscientes de reproduction et de saisir comment des agencements spatiaux "patchwork d'actions humaines imbriquées ", surgissent, se voient
12. J'emprunte ici le terme à des travaux qui, dans les géographies anglo-saxonnes et allemande, ont repris le concept de "perfomativity" à Judith Butler et à ses écrits sur les identités genrées pour penser la construction quotidienne, inconsciente et itérative de la réalité sociale (et spatiale) par la parole et le geste. Selon cette perspective, discours et pratiques actualisent en permanence les normes, composent le réel, le "naturalisent " et assurent sa stabilité (Rose 1999,; Thrift, 2000, ; Scholtmann 2005, ; Strüver, Wucherpfennig 2009).

13. Dans une étape ultérieure d'élaboration de ce travail, je pense travailler les longues durées géoculturelles à partir du concept de " médiance " d'Augustin Berque qui a, depuis Henri Lefebvre, poussé largement plus loin la pensée de la dimension géographique de la condition humaine (Berque, 1990 et 1996). 
14. Je reprends

I'expression que Bernard Debarbieux (2006, p. 347 sq.) mobilise dans son examen critique de ce qu'il appelle le " paradigme territorial ». II cite ici Marie Cuillerai et Marc Abéles (2002, p. 15). réifiées dans les constructions identitaires et «deviennent, dans la foulée, les modérateurs [des] imaginaires, et [des] mémoires. [...] On les regarde; on les garde; ils nous gardent. " (Piveteau, 1995, p. 114). Certains éléments des mécanismes observés à propos du Banat procèdent de cette boucle de reproduction, dans la durée, de constructions territoriales et identitaires. Les habitants construisent une "conscience régionale " articulée à une interprétation de leur histoire et qu'ils projettent dans l'avenir. Les clochers baroques et les morphologies villageoises et urbaines dans le Banat sont enrôlés dans ce double mouvement d'appropriation et d'appartenance territoriales qui motive et fonde le sens des choix individuels et collectifs.

L'erreur consisterait toutefois à mettre les apparitions cartographiques des empires défunts au seul compte de ces mécanismes endogènes, produits en propre par les sociétés régionales, et inscrits dans un long continuum historique. Deux faisceaux au moins de faits observés empiriquement viennent contredire cette lecture. On observe premièrement que les fantômes de nos cartes sont apparus à un moment donné, alors qu'ils étaient invisibles ou peu visibles à des moments antérieurs. La différence régionale observée ne tend pas à se résorber; elle tend au contraire, nous l'avons vu, à s'accroître entre les deux derniers recensements. On observe deuxièmement que les ressorts du chemin spécifique emprunté par les villages olténiens (leur plus grande lenteur à moderniser les maisons et à les doter de pompes à eau que les villages du Banat voisin) ne passe pas - nous l'avons vu plus haut - par des mécanismes d'autoreproduction d'un " triangle magique culture-territoire-identités " (Debarbieux, 2006) ${ }^{14}$ qui les auraient cantonnés dans des identités et des modes de vie plus "traditionnels "; ils procèdent au contraire d'une forme d'autodéni régional qui ne reconnait pas à l'héritage territorial la capacité de donner accès à la modernité souhaitée et qui cherche à rompre avec lui en important de l'extérieur de nouveaux modèles, de nouveau objets et de nouvelles pratiques. Ces deux observations nous mettent sur la piste d'une approche situationniste et pragmatique des mécanismes à l'œuvre. Elles nous invitent avec Bernard Lepetit à ne pas nous limiter "à établir les filiations que supposent les phénomènes auto-entretenus ", et à chercher plutôt à "reconstituer les précédents que chaque moment réactive " (Lepetit, 1995, p. 19).

Expliquons-nous. L'apparition de frontières fantômes dans le moment postsocialiste pourra être comprise, dans cette nouvelle perspective, comme un moment de réactivation d'éléments du passé que l'ordre spatial et politique de la période communiste avait tendu à neutraliser ou à vider de sens et d'intérêt pour les acteurs locaux. La promesse soviétique tournée vers des avenirs qui chantent (bien qu'en grande partie décrédibilisée), était en effet adossée au régime politique qui dictait aux villageois ses voies d'accès au "progrès " et à l'amélioration des conditions matérielles: formation professionnelle, conformité au moins de façade à l'orthodoxie officielle et proximité aux réseaux du Parti. Cette promesse, même discréditée, fournissait des clés de compréhension du présent et guidait les choix des familles. Sa complète disqualification idéologique et politique et le basculement géopolitique des années 1990 a fondamentalement réaménagé le sens de l'expérience vécue et les horizons d'attente en mettant désormais l'initiative individuelle au centre des modèles de réussite et en faisant passer le pronostic favorable au compte des qualités prêtées aux individus et aux sociétés locales. Les proximités ou les similarités avec l'Ouest ont dès lors constitué la meilleure garantie de succès et se sont construites en ressources pour faire face aux nouveaux défis que les acteurs locaux étaient soudain sommés de relever. On le voit, la redéfinition du sens donné à l'Histoire, 
opérée par la disqualification du projet socialiste s'est doublée d'une redéfinition du sens donné aux situations géographiques. C'est à l'intérieur de ces nouvelles coordonnées à la fois temporelles et spatiales que les acteurs font leurs choix domestiques, économiques ou politiques. Le retournement du sens donné à l'expérience du temps historique a redonné sens et valeur à des éléments légués dans les territoires de l'ancienne double monarchie (héritages culturels, fonciers, architecturaux, etc.) que la période socialiste avait discrédités. Il en a réveillé le fantôme en quelque sorte. "Le mort saisit le vif " (Halbwachs, 1997, p. 216).

C'est ici que la métaphore du fantôme nous est utile. Nous ne sommes pas en mesure de démêler dans le discours et les pratiques des acteurs locaux que nous interrogeons et que nous observons ce qui, de l'héritage territorial matériel et social ou bien des potentiels qu'ils lui prêtent et des futurs qu'ils projettent, conditionne leur choix et motive leur agir. La recherche de causalité ne peut exclure ni les croyances, qui sont situées dans la sphère du langage et des représentations, ni les processus historiques et cumulatifs situés dans les apprentissages cognitifs, dans les institutions formelles et informelles ou dans les héritages matériels. L'analyse récuse ici une posture dualiste qui autonomiserait la pensée et l'agir sur le monde à l'égard de sa matière et de sa corporéité. Les acteurs locaux tracent leur devenir à l'intérieur d'un arc de tension qui lie les prophéties géographiques, énoncées dans les cartes mentales, à leur expérience concrète et située.

Au-delà du laboratoire des géographies fantômes du centre-est européen. Vers une nouvelle théorie des aires culturelles

Au-delà des curiosités empiriques que représentent les frontières fantômes repérables dans l'espace centre-est-européen, l'analyse que nous en conduisons permet de reprendre sur un mode rénové la question des longues durées géographiques. Elle montre la voie d'une autre manière de comprendre la stabilité des régions culturelles.

$\mathrm{Au}$ moins deux grands types de métaphores ont généralement guidé la lecture des longues durées géographiques: celui de la "prison de longue durée " (Fernand Braudel), des dépendances du sentier (Paul Pierson) ou des inerties systémiques, qui situent les acteurs et leur espace d'autonomie sur des " cheminements " historiques contraints, dans un système complexe et dense de relations et de structures (Auriac, 1983; Brunet, 2001), établies au fil d'un processus historique plus ou moins continu; celui des " couches sédimentées " ou superposées du "palimpseste " (Rugg, 1985; Schlögel, 2003; Troebst, 2010), de la mémoire informatique (Durand-Dastès, 2001), de la résilience qui met plutôt l'accent sur la succession de périodes, sur les réécritures successives, sur les réemplois des formes dépendantes des périodes antérieures. Les deux types de métaphores ont en commun de chercher les chaînes de causalité dans les logiques endogènes et historiques. Elles tendent à penser les entités culturelles régionales ou les aires civilisationnelles identifiées comme des "êtres géographiques " (Brunet, 2001) dont l'existence perdure, et dont on peut identifier l'origine et suivre le devenir. Selon ce schéma, les caractères régionaux et les aires culturelles existent en soi, avant d'être identifiés par les savoirs géographiques. Nous avons dit la critique dont il a fait l'objet. Au regard de ce débat, nous proposons de travailler à partir de la métaphore du fantôme à l'élaboration d'un concept de fantôme géographique qui permettrait de penser les spatialités culturelles et le rapport temps/espace sur un mode rénové. Cet effort de conceptualisation présente un double intérêt heuristique. 
Premièrement, il permet d'ouvrir le rapport temps/espace/culture - classiquement fondé sur l'accumulation ou sur l'enchaînement historique - au futur et à ses logiques de croyance. Le faisceau serré des actions conduites par une multiplicité d'acteurs qui tissent des territorialités imbriquées et qui produisent un espace géographique différencié, ne se fait pas dans la seule dépendance au passé, elle est aussi dans celle des imaginaires du futur. Ceci n'est pas à confondre avec les effets géographiques que peuvent avoir certaines prophéties autoréalisatrices, certes capables d'orienter les choix de localisation des grands acteurs économiques et politiques ou de simples habitants (Staszak, 2000) mais qui se situent à l'intérieur d'une rationalité et d'un référentiel géographique et historique unique; ici, nous cherchons à faire place à une pluralité de "régimes d'historicité" (Hartog, 2003) et à leur capacité de façonner des régimes territoriaux différents. Nous avons, dans notre analyse, eu recours aux catégories "d'espace d'expérience " et "d'horizon d'attente ». Ces deux catégories construites en miroir, sont reprises à Reinhart Koselleck (1990) qui les a élaborées pour penser le temps historique. R. Koselleck définit l'« espace d'expérience ", comme le "passé actuel, dont les événements ont été intégrés et peuvent être remémorés ". Se rejoignent dans cet espace d'expérience à la fois "l'élaboration rationnelle et des comportements inconscients qui ne sont pas ou plus obligatoirement présents dans notre savoir ". C'est la somme de toutes les expériences concrètes et personnelles ainsi que de l'expérience transmise par les générations précédentes et dans les institutions; l'espace d'expérience assure l'actualité du passé au présent et constitue le rapport de l'individu à son histoire (Koselleck, 1990, p. 311). Précisons que l'historien et théoricien de l'expérience historique ne parle pas de l'espace géographique. Il ne s'y intéresse pas et ne conduit pas davantage d'enquête sur les variations dans l'espace de l'expérience historique. Il fait un usage métaphorique du terme de "Raum " pour dégager la notion d'expérience de sa dimension narrative et la condenser en un tout synchrone au sein duquel les couches du passé sont simultanément présentes, données là, sous différentes formes idéelles, corporelles, institutionnelles et notamment linguistiques. L'expérience est pour lui une connaissance implicite qui se fait habitus aussi bien au niveau individuel qu'au niveau collectif. Nous proposons d'étendre le concept d'espace d'expérience à une dimension non métaphorique. La place qu'il fait aux institutions dans sa définition de l'espace d'expérience peut nous semble-t-il nous y autoriser. Cela suppose de prendre en compte les institutions (formelles et informelles) jusque dans leur dimension spatialisée et de considérer les paysages, les morphologies, interactions et pratiques spatiales, comme des formes ultimes d'institutionnalisation et de matérialisation des expériences collectives. Selon cette perspective phénoménologique, l'espace géographique est ici traité comme une empreinte-matrice des expériences historiques sans cesse réinterprétées et remodelée au fil du temps et, donnée là, au présent des acteurs. Cet élargissement du concept de Koselleck d'espace d'expérience à l'espace géographique pourrait en retour permettre de réintroduire les effets proprement spatiaux de la distance dans le façonnement d'expériences historiques plurielles tant collectives qu'individuelles: permettre de situer l'expérience non seulement dans le décours de l'histoire mais aussi au regard des effets d'éloignement ou de proximité, de la variété des spatialités et des contextes géographiques.

Dans le dispositif de Koselleck, les choix individuels et collectifs se font aussi au regard d'un second volet, celui de "l'horizon d'attente ». L' " attente ", c'est " un futur actualisé, [...][qui] tend à ce-qui-n'est-pas-encore, à ce-qui-n'est-pas-du-champ-de- 
l'expérience, à ce-qui-n'est-pas-encore-qu'aménageable ». "L'espoir et la crainte, le souhait et la volonté, le souci mais aussi l'analyse rationnelle, la contemplation réceptive ou la curiosité - tout cela entre dans sa composition et constitue l'attente. ". À la différence de l'expérience, l'attente ne peut être rapportée à un " espace " et s'inscrit dans un horizon abstrait (Koselleck, 1990, p. 311). L'exceptionnelle richesse heuristique du binôme de l'expérience et de l'attente tient ici à l'interdépendance qui noue ces deux versants du présent des acteurs et c'est ce qui nous intéresse plus particulièrement ici: les acteurs font leurs choix entre une expérience, "saturée de réalité " (Koselleck, 1990, p. 313) et une attente, imprégnée d'imaginaire. L'attente se nourrit de l'expérience (elle la déborde aussi). Inversement l'expérience trouve sens et forme au prisme des attentes (p. 314). Ce dispositif théorique directement inspiré de Koselleck nous permet d'approcher ce que nous pourrions appeler les modalités "vernaculaires " (Jackson, 2003; Besse, 2004) de production des aires culturelles, celles qui se jouent à l'échelon des choix et de l'agir quotidiens des "gens de peu ». C'est chaque fois au présent d'une période particulière que l'on peut saisir la manière dont les sociétés locales redessinent des ensembles géoculturels aux limites mouvantes et entrelacées: chaque fois, les acteurs actualisent, ou au contraire disqualifient, ce qu'ils héritent des configurations du passé en fonction du sens qu'ils leur confèrent rétrospectivement et des défis qu'ils voient venir dans le futur.

L'analyse de régions fantômes permet deuxièmement d'observer que les futurs imaginés ne sont pas seulement portés par l'expérience vécue en propre par les sociétés locales qui se projetteraient vers l'avant à partir de leur seul vécu historique. Ils sont aussi imprégnés des cartes mentales produites à une toute autre échelle par une "géographie de l'Histoire " (Grataloup, 2011) qui nomme les centres et les périphéries, les précurseurs et les suiveurs, distribue les bons et les mauvais points et, ce faisant, dessine une géographie des promesses d'avenir que les sociétés locales ont intériorisées. Les pronostics d'avenir livrés implicitement dans les cartes mentales, participent pleinement de l'élaboration des horizons d'attentes des habitants des régions du monde. On sait que ces géographies mentales ne sont pas immuables. Elles peuvent même s'avérer labiles, sensibles aux mutations des référentiels politiques: la fin de la Guerre froide, l'émergence du grand récit de la mondialisation ou les crises économiques ont été autant de moments de leur réaménagement récent. Ces différentes cartes mentales, construites au cours des deux derniers siècles, sont la transcription spatialisée de grands récits qui demeurent puissamment actifs dans la manière dont les sociétés locales lisent leur propre histoire et imaginent leur avenir. Ces cartes sont habitées de récits et de prophéties d'autant plus efficaces qu'ils sont toujours vécus par les acteurs locaux comme naturels, et demeurent imperméables aux déconstructions savantes, conduites par l'historiographie des dernières décennies.

L'adoption de ces deux thèses qui structurent notre concept du fantôme géographique - (1) les ensembles géoculturels se redessinent sans cesse dans la double dépendance de l'espace d'expérience et de l'horizon d'attente des sociétés, c'est ce qui fait leur historicité ; (2) les horizons d'attentes des acteurs locaux tendent à se conformer aux "destins » que leur assignent les géographies imaginaires dessinées par des savoirs institués - permet d'échapper à l'alternative impossible énoncée en première partie. Elle permet de considérer les deux types de logiques - structurelles ou discursives, que le débat entre Holm Sundhaussen et Maria Todorova avait campées dans des positions opposées - sans les renvoyer dos à dos et en cherchant au contraire 
à les articuler. Elle permet de jeter un regard nouveau sur la construction dans l'espace des différences culturelles. La posture que nous proposons donne les moyens de revisiter la question des longues durées géoculturelles sur la base (paradoxale) d'une forme de présentisme méthodologique qui cherche dans le présent de chaque action (un présent saturé d'expériences et imprégné d'attentes), la compréhension de l'actualisation (ou de l'effacement) de caractères régionaux établis dans le passé.

\section{Références}

Anderson B. (1996). L'Imaginaire national. Réflexions sur l'origine et l'essor du nationalisme. Paris: Éditions La Découverte. $214 \mathrm{p}$.

Auriac F. (1983). Système économique et espace. Paris: Économica, coll. «Geographia », 216 p.

Bachmann-Medick D. (2006). Cultural Turns. Neuorientierungen in den Kulturwissenschaften. Reinbek bei Hamburg: Rowohlt Taschenbuch Verlag, $430 \mathrm{p}$.

BARTH F. (1969). Ethnic Groups and Boundaries. The Social Organization of Culture Difference. Londres: George Allen \& Uwin, $154 \mathrm{p}$.

Becker S.O., Boeckh K., Hainz C., Woessmann L. (2016). « The Empire is dead, long live the empire! Long-run persistence of trust and corruption in the Bureaucracy ». The Economic Journal, vol. 126, nº 590, p. 40-74.

Berque A. (1990). Médiance. De milieu en paysage. Montpellier: Gip Reclus, coll. "Géographiques », $164 \mathrm{p}$.

BeRQue A. (1996). Être humain sur la terre. Paris: Gallimard, coll. "Le Débat», 212 p.

BESSE J.-M. (2003). « Le paysage, entre le politique et le vernaculaire. Réflexions à partir de John Brinckerhoff Jackson ». ARCHES, Association Roumanie des chercheurs francophones en sciences humaines, no 6, p. 9-27. https://halshs.archives-ouvertes.fr/halshs-00113275

BESSE J.-M. (2004). « Le postmodernisme en géographie. Éléments pour un débat ». L'Espace géographique, t. 33, $\mathrm{n}^{0}$ 1, p. 1-5.

Blotevogel H.H. (1996). « Auf dem wege zu einer, theorie der regionalität: Die region als forschungs objekt der geographie ». In BRunn G. (dir.), Region und Regionsbildung in Europa. Konzeptionen der Forschung und empirische Befunde. Baden-Baden: Nomos, p. 44-68.

Brunet R. (dir.)(1990-1996). Géographie Universelle. Paris : Hachette, Belin, GIP Reclus, 10 vol.

BRUnet R. (2001). Le Déchiffrement du monde. Théorie et pratique de la géographie. Paris: Belin, coll. « Mappemonde », $402 \mathrm{p}$.

Collectif (2004). « Débat: Le postmodernisme en géographie. ». L'Espace géographique, t. 33 , $n^{0} 1$, p. 6-37.

Cuilleral M., AbélÈS M. (2002). « Mondialisation: du géo-culturel au bio-politique ». Anthropologie et sociétés, vol. 26, nº 1, p. 11-28.

Darden K., Gzymala-Busse A. (2006). «The great divide: Literacy, nationalism, and the communist collapse ». World Politics, vol. 59, nº 1, p. 83-113.

Debarbieux B. (2006). «Prendre position: réflexion sur les ressources et les limites de la notion d'identité en géographie ». L'Espace géographique, t. 35, n ${ }^{4}$, p. 340-354.

DeSSEMONTET P. (2005). « Révolution orange : la fracture ukrainienne ». EspacesTemps.net. http://www.espacestemps.net/articles/revolution-orange-la-fracture-ukrainienne/

Durand-Dastès F. (2001). "Le temps, la Géographie et ses modèles ». Bulletin de la Société géographique de Liège, t. 40, nº 1, p. 5-13. 
Françols É. (1993). Protestants et catholiques en Allemagne. Identités et pluralisme. Augsbourg 1648-1806. Paris: Albin Michel, coll. «L'Évolution de l'humanité», 392 p.

Glasze G., Füller H., HusseinI de Araujo S., Boris M. (2014). « Regionalforschung in der Geographie und interdisziplinäre areas studies nach dem cultural turn: Eine Einführung ». Geographische Zeitschrift, vol. 102, nº 1, p. 1-6.

Grataloup C. (1996). Lieux d'histoire. Essai de géohistoire systématique. Montpellier: Reclus, coll. «Espaces modes d'emploi », $200 \mathrm{p}$.

GRASLAND C. (1997). «L'analyse des discontinuités territoriales: l'exemple de la structure par âge des régions européennes vers 1980 ». L'Espace géographique, t. 26, nº 4, p. 309-326.

Grataloup C. (2011). Faut-il penser autrement l'histoire du monde? Paris: Armand Colin, coll. «Éléments de réponse», $212 \mathrm{p}$.

Grosfeld I., Zhuravskaya E. (2013). «Persistent effects of empires: Evidence from the partitions of Poland ». Paris School of economics, Working Paper n ${ }^{\circ} 5$. https://halshs.archives-ouvertes.fr/halshs-00795231/document

Halbwachs M. (1997). La Mémoire collective. Paris: Albin Michel, coll. «Bibliothèque de l'évolution de l'humanité», 296 p.

Hartog F. (2003). Régimes d'historicité. Présentisme et expériences du temps. Paris: Éditions Le Seuil, coll. «La Librairie du xxle siècle», 258 p.

HIRSCHHAUSEN B. von (2015). «Phantomgrenzen zwischen Erfahrungsraum und Erwartungshorizont. Konzeptionelle Reflexionen an einem empirischen Beispiel ». In HirchHausen B. von, Grandits H., Kraft C., Müller D., Serrier T. (dir.), Phantomgrenzen. Räume und Akteure in der Zeit neu denken. Göttingen : Wallstein Verlag, p. 84-106.

JACKSON J.B. (2003). À la découverte du paysage vernaculaire. Arles, Versailles: Actes Sud, École nationale supérieure du paysage, $278 \mathrm{p}$.

KoselleCK R. (1990). Le Futur passé. Contribution à la sémantique des temps historiques. Paris: Éditions de l'École des hautes études en sciences sociales, coll. " Recherches d'histoire et de sciences sociales», $334 \mathrm{p}$.

Kraft C. (2015). « Phantomgrenzen und Zeitschichten im Postsozialismus. Ist der Postsozialismus postkolonial? ». In Hirchhausen B. von, Grandits H., Kraft C., Müller D., Serrier T. (dir.), Phantomgrenzen. Räume und Akteure in der Zeit neu denken. Göttingen: Wallstein Verlag, p. 166-190.

Kundera M. (1983). « Un Occident kidnappé ou la tragédie de l’Europe centrale ». Le Débat, nº 27-5, p. 3-23.

Lefebvre H. (1974). La Production de l'espace. Paris: Éditions Anthropos, coll. "Société et urbanisme», $486 \mathrm{p}$.

LemBerg H. (1985). «Zur Entstehung des Osteuropabegriffs im 19. Jahrhundert Vom „Norden“ zum „Osten“ Europas ». Jahrbücher für Geschichte Osteuropas, vol. 33, nº 1, p. 48-91.

LEPETIT B. (dir.)(1995). Les Formes de l'expérience. Une autre histoire sociale. Paris: Albin Michel, coll. «L'Évolution de l'humanité», 337 p.

LöwIS S. von (2015). « Phantom borders in the political geography of East Central Europe: An introduction ». Erdkunde, vol. 69, n² 2, p. 99-106.

MıнĂlLESCU V. (2011). « Comment le rustique vint au village. Modernité domestique et domestication de la modernité dans les campagnes roumaines ». Terrain, n 57 , p. 96-113.

MıHĂllESCU V. (2014). " "Something Nice." Pride houses, post-peasant society and the quest for authenticity ». Cultura, vol. 1, nº 2, p. 83-107.

https://www.pdcnet.org//pdc/bvdb.nsf/purchase?openform \&fp=cultura\&id=cultura_2014_0011_0002_0083_0107\&onlyautologin=true 
Murgescu B. (2010). România si Europa. Acumularea decalajelor economice (1500-2010). Iași: Polirom, $528 \mathrm{p}$.

PEISAKHIN L. (2013). «In history's shadow: Do formal institutions leave a cultural legacy? ». European Political Science Association, 3rd Annual General Conference, Barcelone, 20-22 juin 2013, article 286. http://papers.ssrn.com/sol3/papers.cfm?abstract_id=2224765

Peisakhin L. (2015). "Cultural legacies: Persistence and transmission ». In Schofield N., Caballero G. (dir.), The Political Economy of Governance. Institutions, Political Performance and Elections. Cham : Springer International Publishing, p. 21-39.

Pierson P. (2004). Politics in Time. History, Institutions, and Social Analysis. Princeton, Oxford: Princeton University Press, 196 p.

PiveteAu J.-L. (1995). « Le territoire est-il un lieu de mémoire? ». L'Espace géographique, t. 24, nº 2, p. 113-123.

Prévélakis G. (dir.)(2013). Géopolitique des civilisations. Huntington, 20 ans après. Paris: CNRS Éditions, coll. «Anatoli », n 4,278 p.

Putnam R.D. (1993). Making Democracy Work. Civic Traditions in Modern Italy. Princeton : Princeton University Press, $258 \mathrm{p}$.

Rey V. (1995). «L'Europe centre orientale, un « entre-deux ». In Bailly A., Ferras R., Pumain D. (dir.), Encyclopédie de géographie. Paris: Economica, p. 813-825.

Rey V., Billaut M., Daniel O.Roux M. (1996). «Europes orientales ». In Brunet R., Rey V. (dir.), Géographie Universelle. T. 10 : Europes orientales, Russie; Asie centrale. Paris: Belin, p. 7-206.

Rey V., Groza O., Ianos I., Patorescu M. (2000). Atlas de la Roumanie. Paris: Cnrs-Gdr Libergéo, La Documentation francaise, coll. «Dynamiques du territoire», $168 \mathrm{p}$.

Rey V., Groza O., Ianos I., Patorescu M. (2007, $2^{\mathrm{e}}$ éd.). Atlas de la Roumanie. Paris: CNRS GDRE S4, La Documentation francaise, coll. «Dynamiques du territoire», $208 \mathrm{p}$.

ReY V., Mulatier S., Roth H. (2004). «Élargissement de l'Union européenne: résultats des référendums sur l'adhésion dans les pays d'Europe centrale et orientale ». Cybergeo, http://cybergeo.revues.org/3389

Rose G. (1999). «Performing space ». In Massey D.B., Allen J., Sarke P. (dir.), Human Geography Today. Cambridge: Polity Press, p. 247-259.

RugG D.S. (1985). Eastern Europe. New York: Longman, coll. «World's landscapes», 402 p.

SAID E.W. (1980). L'Orientalisme. L'Orient créé par l'Occident. Paris : Éditions Le Seuil, 392 p.

SChenk B.F. (2002). «Mental Maps. Die Konstruktion von geographischen Räumen in Europa seit der Aufklärung. Literaturbericht ». Geschichte und Gesellschaft, vol. 28, p. 493-514.

SCHLÖGEL K. (2003). Im Raum lesen wir die Zeit. Über Zivilisationsgeschichte und Geopolitik. Munich: Carl Hanser Verlag, $566 \mathrm{p}$.

Schlottmann A. (2005). RaumSprache. Ost-West-Differenzen in der Berichterstattung zur deutschen Einheit. Eine sozialgeographische Theorie. Stuttgart: Franz Steiner Verlag, 343 p.

Schultz H.D. (1980). Die deutschsprachige Geographie von 1800-1970. Ein Beitrag zur Geschichte ihrer Methodologie. Berlin: Selbstverlag des Geographischen Instituts dr Freien Universität Berlin, $478 \mathrm{p}$.

Schultz H.D. (1997). « Räume sind nicht, räume werden gemacht: zur genese "Mitteleuropas" in der deutschen geographie ». Europa Regional, vol. 5, p. 2-14.

http://nbn-resolving.de/urn:nbn:de:0168-ssoar-48375-7

StaRK D., Bruszt L. (1998). Postsocialist Pathways. Transforming Politics and Property in East Central Europe. Cambridge: Cambridge University Press, coll. «Cambridge Studies in comparative politics», $284 \mathrm{p}$. 
StASZAK J.-F. (2000). «Prophétie autoréalisatrice et géographie ». L'Espace géographique, t. 29, $n^{0}$ 2, p. 105-119.

StRÜVER A., WUCHERPFENNIG C. (2009). « Einfühurung: Performativität als diskursive praxis der produktion von realität ». In Glasze G., MatTissek A. (dir.), Handbuch Diskurs und Raum. Theorien und Methoden für die Humangeographie sowie die sozial- und kulturwissenschaftliche Raumforschung. Bielefeld: Transcript Verlag, p. 107-128.

Sundhaussen H. (1999). «Europa balcanica. Der Balkan als historischer raum Europas ». Geschichte und Gesellschaft, vol. 25, n 4, p. 626-653.

SUndhausSen H. (2002). « Was ist Südosteuropa und warum beschäftigen wir uns (nicht) damit? ». Südosteuropa Mitteilungen, vol. 42, nº 5-6, p. 92-105.

Sundhaussen H. (2003). «Der Balkan: Ein plädoyer für differenz ». Geschichte und Gesellschaft, vol. $29, n^{\circ} 4$, p. 608-624.

Thrif N. (2000). « Performance ». In Johston R.J., Gregory D., Pratt G., WatTs M. (dir.), The Dictionary of Human Geography. Oxford: Blackwell Publishers, p. 577.

Todorova M. (1996). «The Ottoman legacy in the Balkans ». In Brown L.C., Imperial Legacy. The Ottoman imprint in the Balkans and the Middle East. New York: Columbia University Press, p. 45-77.

Todorova M. (2002). «Der Balkan als analysekategorie: Grenzen, raum, zeit ». Geschichte und Gesellschaft, vol. 28, n 3, p. 470-492.

Todorova M. (2003). « Historische vermächtnisse als analysekategorie. Der fall Südosteuropa ». In Kaser K., Gramshammer-Hohl D., Pichler R. (dir.), Europa und die Grenzen im Kopf. Klagenfurt: Wieser Verlag, p. 227-252.

Todorova M. (2007). Die Kategorie Zeit in der Geschichtsschreibung über das östliche Europa. Leipzig: Oskar-Halecki-Vorlesung, $50 \mathrm{p}$.

Todorova M. (2011). Imaginaire des Balkans. Paris: Éditions de l'École des hautes études en sciences sociales, coll. «En temps \& lieux», 352 p.

TROEBST S. von (2010). ",,Geschichtsregion” Historisch-mesoregionale Konzeptionen in den Kulturwissenschaften ». Europäische Geschichte Online (EGO).

http://ieg-ego.eu/de/threads/theorien-und-methoden/geschichtsregion/stefan-troebst-geschichtsregion

Wardenga U., Miggelbrink J. (1998). «Zwischen realismus und konstruktivismus: Regionsbegreiffe in der geographie und anderen humanwissenschaften ». In Wollersheim H.-W., Tzschaschel S., MiddelL M. (dir.), Region und Identification. Leipzig : Leipziger universitätsverlag, p. 33-46.

WeichHaRt P. (1996). «Die Region - Chimäre, artefakt oder strukturprinzip sozialer systeme? »In Brunn G. (dir.), Region und Regionsbildung in Europa. Konzeptionen der Forschung und empirische Befunde. Baden-Baden: Nomos, p. 25-43.

WERLEN B. (1995a). Sozialgeographie alltäglicher Regionalisierung. Band 1: Zur Ontologie von Gesellschaft und Raum. Stuttgart: Franz Steiner Verlag, 262 p.

WERLEN B. (1995b). « Von der Regionalgeographie zur Sozial-kulturgeographie alltäglicher Regionalisierungen ». In Benno Werlen, S. Wälty (dir.), Kulturen und Raum. Chur (Suisse): Ruegger, p. 99-118.

WERLEN B. (2004). « Géographie culturelle et tournant culturel ». Géographie et cultures, n 47, p. 7-27.

Wolff L. (1994). Inventing Eastern Europe. The Map of Civilization on the Mind of the Enlightenment. Stanford: Standford University Press, $436 \mathrm{p}$.

ZARYCKI T. (2000). « Politics in the periphery: Political cleavages in Poland interpreted in their historical and international context ». Europe-Asia Studie, vol. 52, n ${ }^{\circ}$, p. 851-873.

ZARYCKI T. (2015). « The electoral geography of Poland: Between stable spatial structures and their changing interpretations ». Erdkunde, vol. 69, nº 2, p. 107-124. 